\title{
In-situ ETEM observation of intergranular oxidation of copper
}

Xianhu Sun ${ }^{1}$, Richard Garza ${ }^{2}$, Xiaobo Chen ${ }^{3}$, Meng $\mathrm{Li}^{4}$, Stephen House ${ }^{4}$, Wissam Saidi ${ }^{2}$, Judith Yang ${ }^{2}$ and Guangwen Zhou ${ }^{1}$

${ }^{1}$ Binghamton University, United States, ${ }^{2}$ University of Pittsburgh, United States, ${ }^{3}$ Binghamton University, Port Jefferson Station, New York, United States, ${ }^{4}$ University of Pittsburgh, Pittsburgh, Pennsylvania, United States

Grain boundaries (GBs) are planar defects and ubiquitous within polycrystalline materials. Such planar defects affect various physical and chemical properties of materials. This is typified by the preferential intergranular oxidation of metals, which results in the oxide penetration along grain boundaries. Such localized oxide growth can act as stress concentrators to initiate cracking at these regions, leading to spalling of the oxide layer ${ }^{1}$. On the other hand, the effect of grain boundaries is also found beneficial. It was observed that grain boundaries intersecting the alloy surface can aid in the development of a more protective oxide at their adjacent areas during the initial stages of oxidation of $\mathrm{Ni}-\mathrm{Cr}$ alloys by suppressing the oxide penetration while promoting its lateral spread to cover the whole alloy surface ${ }^{2}$. Therefore, these seemingly contradictory roles of grain boundaries may be rooted in the differences in the early-stage oxidation behavior that can exert a strong influence on the microstructure development of the oxide scale in steady-state oxidation.

Unfortunately, experimentally probing grain-boundary oxidation has been a major challenge, mainly because of the spatially localized nature of grain boundaries. The overall reaction kinetics during the initial stages of oxidation of a metal surface containing grains and emerging GBs are complex, due to the convolution of different kinetic factors such as the nucleation and growth of oxide islands on grains as well as at the GBs. Most averaging techniques measuring the oxidation kinetics cannot separate the reactions at these different regions of the surface. Fundamental understanding of grain-boundary oxidation dynamics not only requires resolving the local structure at the atomic scale but also the ability to capture this structural evolution in real time and under the oxidation conditions. Environmental transmission electron microscopy (ETEM) is capable of overcoming this challenge by providing temperature-, time-, and pressure-resolved imaging of gas-surface reactions by introducing a reactive gas to the sample while simultaneously monitoring the structural evolution at the atomic scale. Herein, we employ ETEM to atomically elucidate the effect of grain boundaries on the early-stage oxidation of copper.

Fig. 1a-c is time-resolved plane-view TEM images showing the faster growth rate of $\mathrm{Cu}_{2} \mathrm{O}$ islands at the GB than that on the surface area away from the GB. The alignment of oxide islands (with the round shape) along the grain boundary suggests that, overall, the grain boundary is the preferred site for the nucleation of oxide islands in contrast to the grain surface where oxide islands (with triangle shape) are nucleated randomly. The low-magnification, morphological overview of the oxide formation is complemented by in-situ HRTEM imaging of the atomic process leading to the preferential grain boundary oxidation, as shown in Fig. 1d-i. Fig. $1 \mathrm{~d}$ is an HRTEM image showing a grain boundary composed of two $\mathrm{Cu}$ grains. As shown by the inset diffractograms in Fig. 1d, grain 1 has the [100] zone axis with respect to the incident electron beam and a (010) side facet whereas grain 2 has the [110] zone axis and a (1-12) side facet. Fig. 1e is a zoom-in TEM image of the white dashed square in Fig. 1d showing the (011)/(-111) matching of crystal planes of the two grains. Upon the oxygen exposure, the (100) side facet of grain 1 near the GB develops into the missing-row restructuring in which every fourth row of $\mathrm{Cu}$ atoms is missing ${ }^{3}$. By contrast, the (1-12) facet of grain 2 adjacent to the GB undergoes lattice expansion from the surface to the subsurface region. This indicates that the surface areas adjacent to the GB are more prone to oxygen attack. Particularly, the more open surface structure, i.e., the (112) facet of grain 2 , shows significant oxygen uptake by the subsurface, as evidenced by the observed lattice expansion (Fig. 1f-g). As marked by the sky dashed lines in Fig. 1f-i, the moire fringe contrast becomes visible, starting from the GB and propagating laterally on grain 1 and along the GB. This indicates the nucleation and 
growth of an oxide island (labeled as oxide 1) on grain 1 in the area adjacent to the GB. During the same time period, the oxygenated region of the (1-12) facet of grain 2 transforms into the $\mathrm{Cu}_{2} \mathrm{O}$ (200) lattice spacing (labeled as oxide 2 in Fig. 1h-i). The absence of appreciable moire fringe contrast in the planar surface region (with the (110) surface termination) of grain 2 indicates its higher oxidation resistance. These in-situ TEM observations provide the microscopic detail of the GB-assisted heterogeneous nucleation and growth of $\mathrm{Cu}_{2} \mathrm{O}$ islands. Correlated atomistic modeling will be performed to elucidate the effect of GBs on surface oxidation including oxygen adsorption and mass transport. 


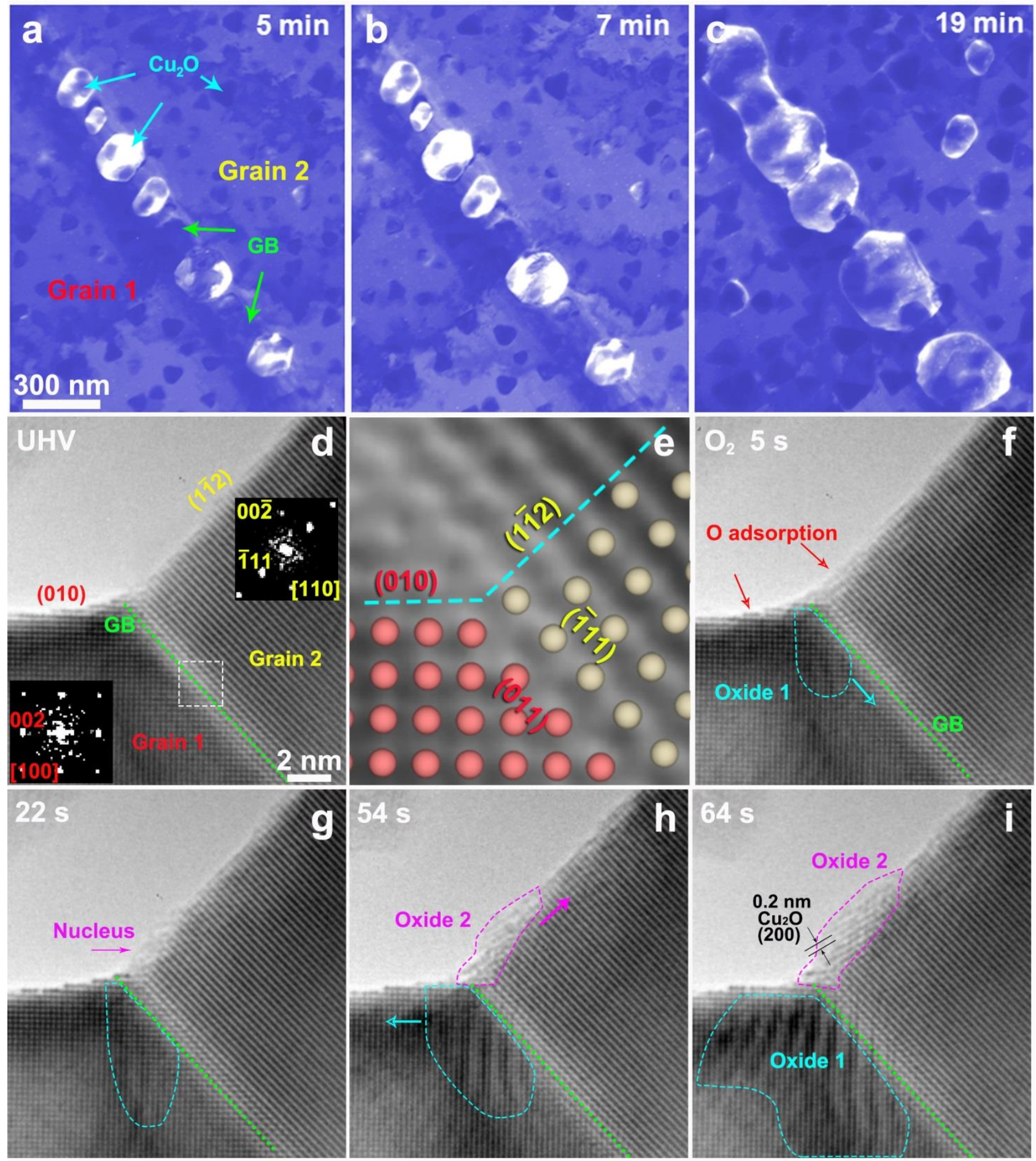

Figure 1. Figure 1. (a-c) Time-resolved bright-field low-magnification TEM images showing the preferred nucleation and growth of $\mathrm{Cu} 2 \mathrm{O}$ islands along the grain boundary during the $\mathrm{Cu}$ oxidation at $\mathrm{pO} 2 \approx 1.3 \mathrm{~Pa}$ and $\mathrm{T} \approx 400{ }^{\circ} \mathrm{C}$. (d) HRTEM image of pristine GB formed by the [100] and [110] oriented Cu grains (insets are the diffractograms of the two grains). (e) Enlarged view of the GB region marked with the white dashed square in (d) showing the lattice planes on each side of the $\mathrm{Cu}$ grains. (f-i) Time-sequenced HRTEM image snapshots showing the preferred nucleation and growth of $\mathrm{Cu} 2 \mathrm{O}$ islands adjacent to the $\mathrm{GB}$. 


\section{References}

1. Molins, R. et al. Acta Mater. 45, 663-674, (1996).

2. UI-Hamid, A. Oxid. Met. 57, 217-230, (2002).

3. Li, L. et al. Phys. Rev. Lett. 108, 176101, (2012).

4. This work was supported by the U.S. Department of Energy, Office of Basic Energy Sciences, Division of Materials Sciences and Engineering under Award No. DE-SC0001135. 